\title{
A General control mechanism of energy flow in the excited state of polyenic biochromophores
}

\author{
Tiago Buckup, ${ }^{a}$ Jürgen Hauer, ${ }^{b}$ Judith Voll, ${ }^{c}$ Regina Vivie-Riedle ${ }^{c}$ \\ and Marcus Motzkus*a
}

\author{
Received 8th March 2011, Accepted 24th March 2011 \\ DOI: 10.1039/c1fd00037c
}

\begin{abstract}
Quantum dynamics in photobiology is a highly controversial subject of modern research. In particular, the role of low-frequency vibrational coherence of biochromophores has been intensely discussed. Coherent control of polyenic chromophores, like carotenoids and retinoids, has been showing that the manipulation of such low frequency coherences may play a crucial role in the evolution of excited population and therefore in the efficiency of photosynthesis. However, no precise control mechanism has been derived. In order to clarify this open question, we combined quantum dynamical modelling with a sensitive experimental technique, namely Pump-Degenerate Four Wave Mixing (PumpDFWM). In this work we investigate in detail the internal conversion channel of $\beta$-carotene, an important polyenic chromophore, under multipulse excitation and focus on the role of the non-adiabatic coupling between excited-state potentials and the internal energy loss. Our control mechanism is based on the interference between wavepackets in the excited state, which leads to a transient evolution of the vibrational population dependent on the relative phase between excitation sub-pulses. Such a transient evolution can affect the branching ratio between competing channels in the excited state. Therefore, our results are able to rationalize pulse shapes found in a whole class of coherent control experiments involving polyenic biochromophores, like in light harvesting complexes and in bacteriorhodopsin.
\end{abstract}

\section{Introduction}

Understanding ultrafast energy flow in the excited state of biological chromophores is an important step in the technological utilization of photosynthesis. The efficiency of the transformation of light into chemical energy is mainly determined by the competition between the ultrafast energy loss and reactive channels. ${ }^{1,2}$ In this regard, intramolecular deactivation processes in the excited reactive states of donor molecules are not desirable and should be identified and possibly suppressed for an efficient photosynthesis.

The identification, however, of such deactivation and reactive channels in biomolecules and how they interplay to make photobiological processes highly efficient are still a challenge in time-resolved spectroscopy. ${ }^{3-5}$ Nature through evolution has been exploiting efficient energy transfer mechanisms by selecting ultrafast processes

\footnotetext{
"Physikalisch-Chemisches Institut, Ruprecht-Karls-Universität Heidelberg, Im Neuenheimer Feld 229, D-69120 Heidelberg, Germany.E-mail: marcus.motzkus@pci.uni-heidelberg.de; Fax: +49 6221 54-8730; Tel: +49 6221 54-8726

${ }^{b}$ Faculty of Physics, University of Vienna, Strudlhofgasse 4, 1090 Vienna, Austria

'Department Chemie, Ludwigs-Maximilian-Universität München, Butenandt-Strasse 11, D-81377 München, Germany
} 
between acceptor and donor chromophores to minimize the effect of interatione Online with the bath. Unfortunately, such a short time scale leads to a very narrow experimental time window where the desired dynamics can be detected. An additional challenge is posed by the transition dipole moments of the involved states: often reactive excited states show strong light absorption from ground state but none or weak excited state absorption. Such weak transition dipoles can make the probing of the respective reactive states particularly demanding. Furthermore, even when these issues are not the main concern or can be avoided, the ultrafast dynamics of photobiological processes are often difficult to disentangle: Transient data may not just contain contributions from degrees of freedom decisive for the reaction itself, but may also include contributions from, e.g., spectator vibrational modes or unconnected energy relaxation.

A modern method to circumvent the issues discussed above and, therefore, assist the investigation of the initial ultrafast steps of photosynthesis is based on the combination of time-resolved spectroscopy and tailored excitation. ${ }^{6-12}$ In this field, specially shaped laser fields can select or suppress a transient spectral signature, leading potentially to the identification of all internal degrees of freedom directly involved in an energy transfer reaction.

In this context, the ultrafast energy transfer and losses in biological complexes involving a main chromophore with polyenic structural design are still intensely debated. A major point regards the role of reactive vibrational modes. The excitation of natural ${ }^{9,13}$ as well as artificial ${ }^{14}$ light harvesting complexes with different carotenoids showed that deactivation and, in one case, energy transfer can be controlled with tailored pulses in form of a sequence of pulses (multipulse excitation-see Table 1 for optimal and anti-optimal sub-pulse separations). More recently, ${ }^{15}$ an independent experiment on the isomerization of retinal showed a similar dependence on the multipulse excitation. The optimal pulse shapes obtained in these control experiments suggested a general mechanism where low

Table 1 Coherent control on polyenic biochromophores: Optimal results are for optimization of energy transfer over internal conversion on the donor chromophore. Frequency values in $\mathrm{cm}^{-1}$ are obtained from the sub-pulse separation in the optimal or anti-optimal multipulse shape. $N$ is the number of conjugated double bonds

\begin{tabular}{lccc}
\hline System & $\begin{array}{c}\text { Natural Light Harvesting } \\
\text { Complex LH2 } 2^{13}\end{array}$ & $\begin{array}{c}\text { Artificial Light } \\
\text { Harvesting } \\
\text { Complex }^{14}\end{array}$ & Bacteriorhodopsin $^{15}$ \\
\hline Chromophore & $\begin{array}{c}\text { Rhodopin glucoside } \\
(N=11)\end{array}$ & $\begin{array}{c}\beta \text {-carotene derivative } \\
(N=10)\end{array}$ & $\begin{array}{c}\text { All } \text { trans-retinal }_{(N=6)} \\
(N=6)\end{array}$
\end{tabular}

Chromophore structure
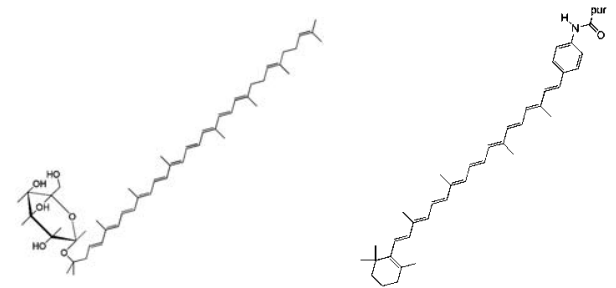

Sub-pulse

N.A.

$300 \mathrm{fs}$

$145 \mathrm{fs}$

separation

$\left(110 \mathrm{~cm}^{-1}\right)$

$\left(230 \mathrm{~cm}^{-1}\right)$

optimal

Sub-pulse

$210 \mathrm{fs}$

$200 \mathrm{fs}$

$215 \mathrm{fs}$

separation

$\left(160 \mathrm{~cm}^{-1}\right)$

$\left(166 \mathrm{~cm}^{-1}\right)$

$\left(155 \mathrm{~cm}^{-1}\right)$

anti-optimal 
frequency modes play a crucial role in the evolution of excited population. Fiteowerende Online the dynamical details of the excitation mechanism remain unclear. Historically, multipulse excitation has been interpreted in a "child-on-a-swing" picture, where the vibrational wavepackets temporally in phase with the sub-pulse separation will be promoted while out-of phase vibrational wavepackets will be suppressed. ${ }^{16}$ In spite of this mechanistic explanation, this model misses an important point: Although out-of-phase vibrational wavepackets are suppressed by an appropriately spaced multipulse, it still bears the same linear spectrum as a Fourier-limited pulse. Therefore, the respective vibrational levels are still populated even if no wavepacket is formed. ${ }^{17,18}$ This is an important aspect given that energy flow in the excited state will take place even if vibrational coherence is weak.

Another open question concerns the electronic state of origin of the observed wavepacket. Via impulsive stimulated Raman scattering, a sufficiently broadband excitation pulse may generate wavepackets either in the electronic excited- or in the ground-state. In the case of a multipulse excitation, as in control experiments for polyenic chromophores, this can lead to different interference effects between the wavepackets generated by each sub-pulse of the sequence because Franck-Condon modes may have different frequencies in each potential surface. Therefore, the "child-on-a-swing" picture does not give any mechanistic explanation for how the interference between these wavepackets may lead to a modulation of energy transfer or loss in the excited-state. Moreover, the nature of the interplay between excited state lifetime and sub-pulse separation remains elusive. Sub-pulse separations much longer than excited-state population lifetime or the electronic coherence time should not have any effect on the molecular dynamics since the system already relaxed before the next sub-pulse arrives at the sample. In situations where strong control over population loss/energy transfer is observed but the sub-pulse separation is on the order of excited population or coherence time, it is not apparent whether the "child-on-a-swing" picture is applicable as it fails to describe the quantum nature of the process.

In order to address this fundamental question about the control of energy flow in biochromophores, we combined quantum dynamical modelling with a very sensitive experimental technique, namely Pump-Degenerate Four Wave Mixing (PumpDFWM $).{ }^{19-23}$ Within this method, the multi-beam DFWM acts as a Probing Sequence (PS) which is free of ground state contributions since Pump-DFWM exploits excited state resonances missing in the static absorption spectrum. In this work we investigate in detail the internal loss channel of $\beta$-carotene, an important polyenic chromophore, under multipulse excitation and focus on the role of the non-adiabatic coupling between excited-state potentials and the internal energy loss.

\section{Experimental details}

\section{Theoretical approach ${ }^{24}$}

$\beta$-carotene, and carotenoids in general, present two low-lying excited states, namely $\mathrm{S}_{2}$ and $\mathrm{S}_{1}$ states. $^{3}$ The $\mathrm{S}_{2}$ state is the first one-photon allowed electronic state. In nature and artificial systems, the competition between $S_{2}-S_{1}$ relaxation and the energy transfer between the $\mathrm{S}_{2}$ state and acceptor molecules (e.g. (bacterio-) chlorophylls or purpurins) is a decisive step for the photosynthetic efficiency. While the energy transfer is described as a dipole-dipole interaction (Förster energy transfer), the mechanism of internal relaxation is still not fully understood. In spite of the controversial presence of additional electronic states between $S_{2}$ and $S_{1}$ states, we have chosen to describe the coupling between $S_{2}$ and $S_{1}$ states without additional excited electronic states. ${ }^{25}$ The validity of such a choice will be discussed later.

In order to describe the excited state dynamics on $S_{2}$ surface in the context of wavepacket tailoring, two representative modes $\left(x=178 \mathrm{~cm}^{-1}\right.$ - the electronic coupling process driving mode, and $y=1157 \mathrm{~cm}^{-1}$-a fast spectator mode) out of 
a ground state normal mode analysis (DFT/b3lyp, 6-31G*) of $\beta$-carøteneArace Online selected to set up the Hamiltonian in the diabatic representation:

$$
H(x, y)=T(x, y)+\left(\begin{array}{ccc}
S_{0}(x, y) & 0 & \varepsilon(t) \mu_{02} \\
0 & S_{1}(x, y) & C_{21}(x, y) \\
\varepsilon(t) \mu_{02} & C_{21}(x, y) & S_{2}(x, y)
\end{array}\right)
$$

Included are the three electronic potential energy states (PES) of $\beta$-carotene $\left(\mathrm{S}_{2}, \mathrm{~S}_{1}\right.$ and $\mathrm{S}_{0}$ ), the transition dipole moment $\mu_{02}$ between $\mathrm{S}_{0}$ and $\mathrm{S}_{2}$ states and the non-adiabatic coupling element $\mathrm{C}_{21}(x, y)$. The intersection between $\mathrm{S}_{2}$ and $\mathrm{S}_{1}$ states, defined by the choice of their displacement parameters, builds the centre of the Gaussian shaped coupling element $C_{21}$. Finally, the simulations are performed by solving the open system Liouville von Neumann equation:

$$
\dot{\rho}(t)=L \rho(t)=-i[H, \rho(t)]+L_{D}(\rho(t))
$$

Solvent effects are described by the dissipative part of Lindblad form introducing vibrational relaxation times of $T_{1 \mathrm{v}}=5 \mathrm{ps}$, pure vibrational dephasing times of $T_{2 \mathrm{v}}=$ $8 \mathrm{ps}$ and pure electronic dephasing times of $T_{2 \mathrm{e}}=600 \mathrm{fs}$ for the appropriate fundamental transitions. Dissipation rates for higher transitions inside one mode are assumed to be proportional to the vibrational quantum number respective the energetic distance. To simulate the fast internal vibrational redistribution (IVR) processes on $\mathrm{S}_{1}$, we introduced an additional fundamental vibrational relaxation time of $T_{1, \mathrm{IVR}}=20 \mathrm{fs}$ deduced from the experimentally observed exponential rise of the DFWM signal intensity. Inclusion of $T_{1, \mathrm{IVR}}$ leads to an effective electronic dephasing time of $200 \mathrm{fs}$ between the two excited states $S_{2}$ and $S_{1}$.

\section{Experimental setup}

In order to simulate/replicate the control experiments in polyenic chromophores with multipulse excitation we combined an open-loop control setup with the

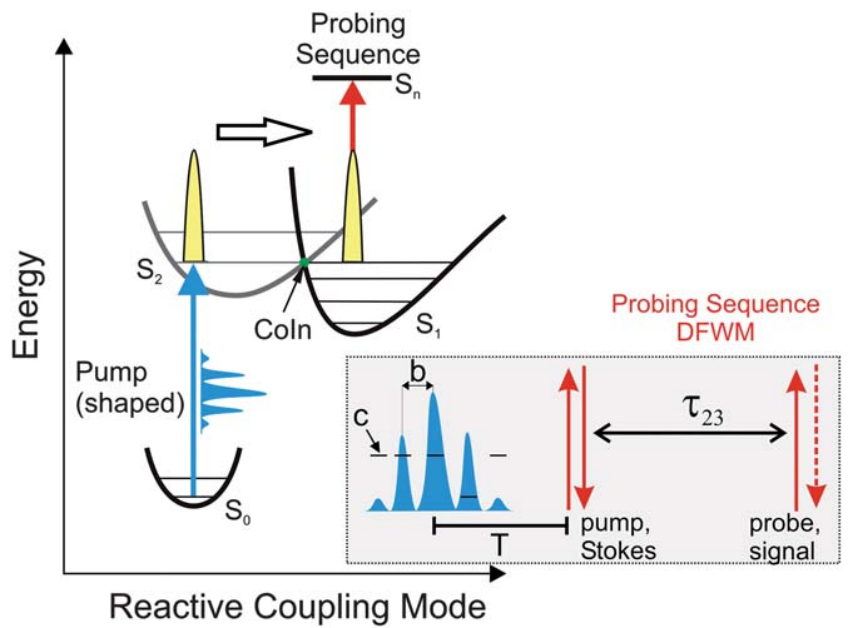

Fig. 1 Potential energy surfaces describing the early photophysics of $\beta$-carotene in solution. The dynamics are defined by a conical intersection (CoIn). The employed excitation scheme involves a pump pulse (blue), phase modulated for coherent control, and a probing sequence (red) used for detection purposes only. Inset shows schematically the sub-pulse separation $b$ and the phase parameter $c$. The initial pump pulse is tailored with a sinusoidal phase. After a delay $T$, a degenerate four-wave-mixing is used as probe sequence. The delay between pump and Stokes was kept at 0 while the delay $\tau_{23}$ was $170 \mathrm{fs}$. 
Pump-DFWM technique. In the Pump-DFWM experiment a tailored punaptote Online (Initial Pump (IP)) pre-excites $\beta$-carotene in the Franck-Condon (FC) region of the $\mathrm{S}_{2}$ electronic state. The relaxation dynamics is probed at a delay $T$ with a probing sequence (PS) which consists of three beams (pump/Stokes/probe) forming a timeresolved degenerate four-wave mixing (DFWM) scheme (Fig. 1). The probe pulse of this FWM sequence is independent and can be delayed $\left(\tau_{23}\right)$ with respect to the timely coincident pump and Stokes pulses $\left(\tau_{12}=0\right)$. The use of the DFWM sequence as probe instead of a single pulse, like in transient absorption (TA), allows the detection of small population variations in the excited state. DFWM, as a backgroundfree nonlinear time-resolved technique, shows a quadratic dependence on the population variation in the excited state $\left(\Delta n^{2}\right)$. A single probe pulse as in TA is not as sensitive to signal variations as the DFWM method because it shows a linear dependence on the population variation $(\Delta n)$.

The initial Pump and DFWM sequence were generated using two separate single staged non-collinear parametric amplifiers (nc-OPA). The initial pump was centred around $510 \mathrm{~nm}$ with a temporal pulse width of $15 \mathrm{fs}$ as determined by an autocorrelator. The DFWM sequence was obtained by splitting the output of another ncOPA into three parts of equal intensity. The spectrum of the DFWM sequence was centred around $560 \mathrm{~nm}$ with duration of $13 \mathrm{fs}$. Before interacting with the sample, the beams were focused by a concave mirror with $30 \mathrm{~cm}$ focal length, resulting in a beam radius of $50 \mu \mathrm{m}$ in the focus. The DFWM signal was detected by a photomultiplier after an interferometric filter centred at $610 \mathrm{~nm}$. Average excitation energies were $40 \mathrm{~nJ}$ for the initial pump $\left(1.3 \times 10^{15}\right.$ photons $\left.\mathrm{cm}^{-2}\right)$ and below $10 \mathrm{~nJ}$ $\left(3.5 \times 10^{14}\right.$ photons $\left.\mathrm{cm}^{-2}\right)$ for the pulses in the DFWM-sequence, which ensured a linear regime of excitation. At these experimental conditions, the initial pump promoted less than $4 \%$ of the molecules to the $S_{2}$ state. Fig. 2 shows the absorption of $\beta$-carotene dissolved in cyclohexane and the experimental excitation spectra. There is no spectral overlap between the linear absorption spectrum and the DFWM spectrum. The DFWM spectrum is resonant with the excited state $\mathrm{S}_{1}-\mathrm{S}_{n}$

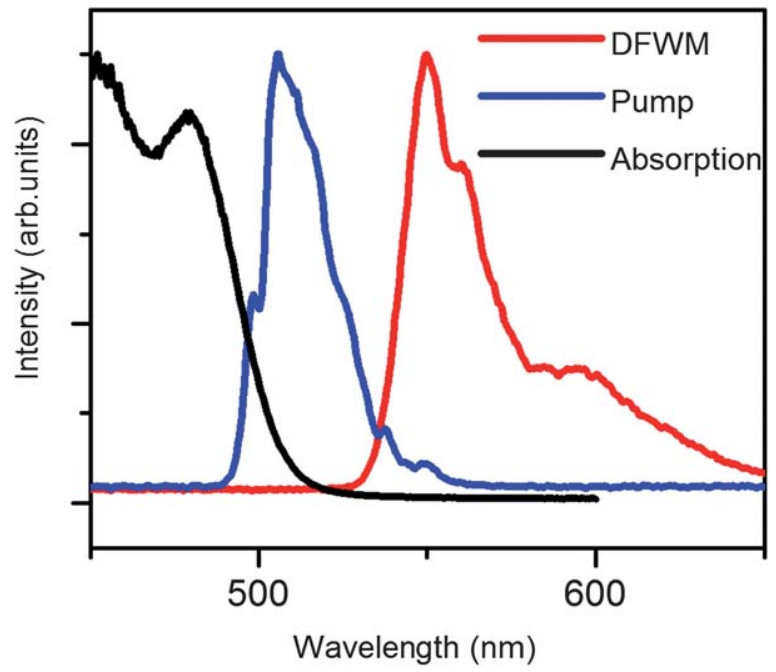

Fig. $2 \beta$-Carotene absorption and Pump-DFWM excitation spectra. The spectrum of the initial pump is near resonant with the ground-state absorption spectrum of $\beta$-carotene. The DFWM, on the other hand, is not resonant with any absorption of the ground-state: Its spectrum is solely resonant with the excited-state absorption of the $\mathrm{S}_{1}$ state, which has a maximum at about $550 \mathrm{~nm}$ in cyclohexane. 
absorption (not shown), and, therefore, probes the arrival of populationiedireatlye Online after the non-adiabatic coupling between the $S_{2}$ and $S_{1}$ states.

In our open-loop control approach, a pure phase modulation is exerted on the initial pump pulse. Its spectral phase $\Phi(\omega)$ is manipulated via a spatial light modulator (SLM) with 128 pixels in a 4f-arrangement. Application of $\Phi(\omega)=$ $a \sin (b \omega+c)$ yields a well defined pulse train. ${ }^{16,26,27}$ The parameter $a$ is kept fixed at 1.23 and parameter $b$ defines the sub-pulse separation. Note that the parameter $c$ defines the relative phase between the sub-pulses as shown in Fig. 3. The absolute phase of each sub-pulse is not controlled in this experiment. In all measurements, a non-zero $T$-delay between the tailored initial pump pulse and DFWM sequence was chosen to avoid the shaping window. ${ }^{28}$ Since the delay $T$ is defined between the centre sub-pulse of the multipulse sequence and the pump/Stokes arrival time, a $T$ delay over $112 \mathrm{fs}(T>2 b)$ guarantees probing outside the shaping window in the case of $b=56 \mathrm{fs}$.

\section{Results}

\section{Experiment}

Usually, in the Pump-DFWM technique, DFWM transients are measured by scanning the probe delay $\tau_{23}$ for different delays $T$ between initial pump and the DFWM sequence. ${ }^{21-23}$ In what follows, the probe delay was kept fixed while the initial pump shaping was varied by tailoring the parameter $c$. In Fig. 4, we present these results in a 2D graph, where the Pump-DFWM signal along the $T$-axis was plotted for different values of the parameter $c$. In this way, it allows the investigation of the effect of excitation phase on the internal conversion between $S_{2}$ and $S_{1}$ states. Fig. 4 (a) depicts such a kind of Pump-DFWM signal. The excitation pulse was shaped into a pulse train with a sub-pulse spacing $b=56 \mathrm{fs}$ and varying the parameter $c$ between the sub-pulse. This value of parameter $b$ guarantees three well separated sub-pulses within the life time of $S_{2}(180 \mathrm{fs})$. Along the $y$-axis of Fig. 4 (a), the parameter $c$ between the sub-pulses is varied between 0 and $2 \pi$. The signal increases exponentially from cold colours (low intensities) to hot colours (high intensities) along the $T$-axis. This is due to the fact that the population on $\mathrm{S}_{2}$ relaxes towards the $S_{1}$ entering the Franck-Condon region where we probe the dynamics with the DFWM sequence. Fig. 4 (a) shows that the DFWM signal can be modified by the parameter $c$. In other words, the flow of population from $S_{2}$ state into $S_{1}$ state can be controlled, i.e., can be delayed or speed up by the correct choice of parameter c. The white dotted line at $T=270 \mathrm{fs}$ in Fig. 4 (a) exemplifies it (shown in Fig. 4 (b): While the population for e.g. $c=3 / 2 \pi$ has almost reached its maximum, the
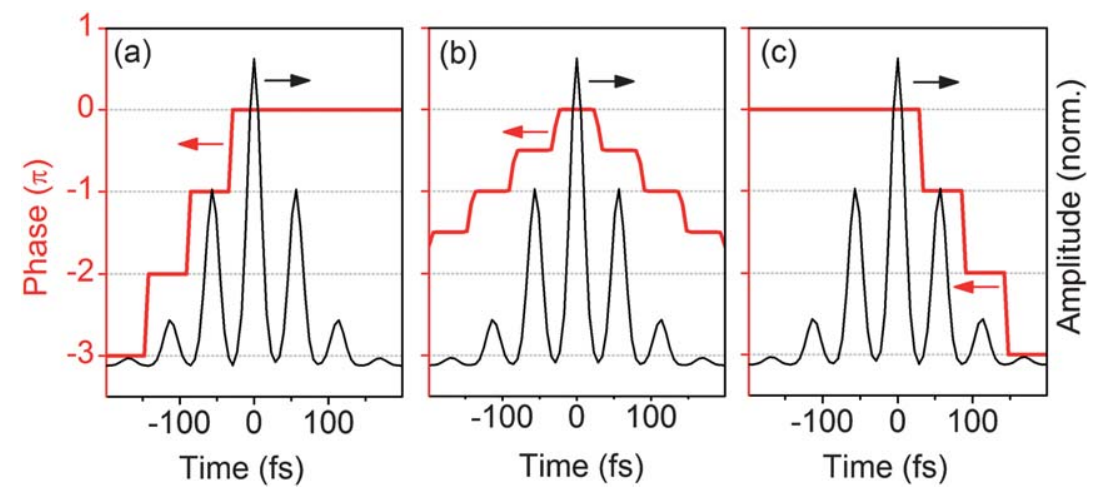

Fig. 3 Simulation of the effect of parameter $c$ on the relative phase between the sub-pulses generated by a sinusoidal phase shaping ( $b=56 \mathrm{fs}$ ). (a) $c=0 \mathrm{rad}$. (b) $c=\pi / 2 \mathrm{rad}$. (c) $c=\pi \mathrm{rad}$. 

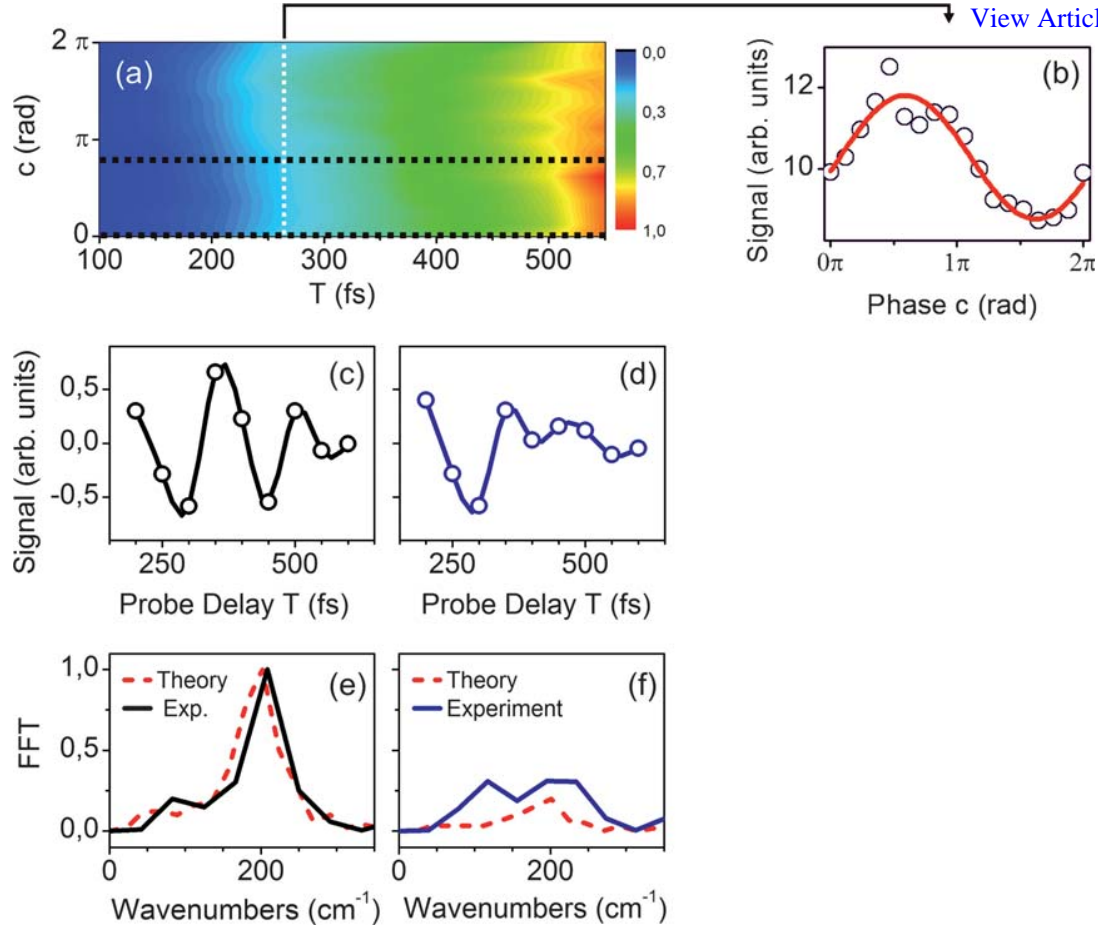

Fig. 4 (a) Pump-DFWM signal at $\tau_{23}=170 \mathrm{fs}$ for various values of $T$ and the excitation parameter $c$. (b) Signal at about $\mathrm{T}=270 \mathrm{fs}$ at different values of parameter $c$. (c) and (d) show the oscillatory component obtained after subtraction of the slow non-oscillatory exponential rise time for $c=0 \mathrm{rad}$ and $2.3 \mathrm{rad}$, respectively. (e) and (f) show the respective Fourier transformations for experimental and simulation data.

population for e.g. $c=1 / 2 \pi$ is still rising. Eventually, independently of the choice of parameter $c$, the whole population excited from the $\mathrm{S}_{0}$ into the $\mathrm{S}_{2}$ will reach the cold $\mathrm{S}_{1}$ state at $T$ delays greater than $600 \mathrm{fs}$. This can be easily understood, since the excited-state population in $\beta$-carotene, as a closed system (i.e. without energy transfer), must relax back to ground-state via the $S_{1}$ deactivation channel. Parallel channels like fluorescence are present but are much less efficient than the ultrafast internal relaxation $\mathrm{S}_{2}-\mathrm{S}_{1}$.

The sensitive DFWM probing sequence is also able to unravel an oscillatory component on the rise of the signal along the $T$-axis. This component can be clearly observed when the slow non-oscillatory exponential rise time is subtracted. This is demonstrated for two values of the parameter $c$ (traced lines at Fig. 4 (a)). The two oscillatory components for $c=0 \mathrm{rad}$ and $2.3 \mathrm{rad}$ are shown at Fig. 4 (c) and (d), respectively. A Fourier transformation of the signal depicted in Fig. 4 shows a clear peak around $200 \mathrm{~cm}^{-1}$ (Fig. 4 (e)), which is not observed for the other $c=2.3 \mathrm{rad}$ (Fig. 4 (f)).

\section{Simulation}

The simulations show that the composition and phase of the wavepacket depends strongly on the parameters of the exciting multipulse. We observe that partial wavepackets, differing in phase and vibrational components, can be generated by each sub-pulse. The choice of $b$ defines the phase difference and therewith the number of induced sub wavepackets. Defining $T_{\text {vib }}$ as the oscillation period of the 
(a)
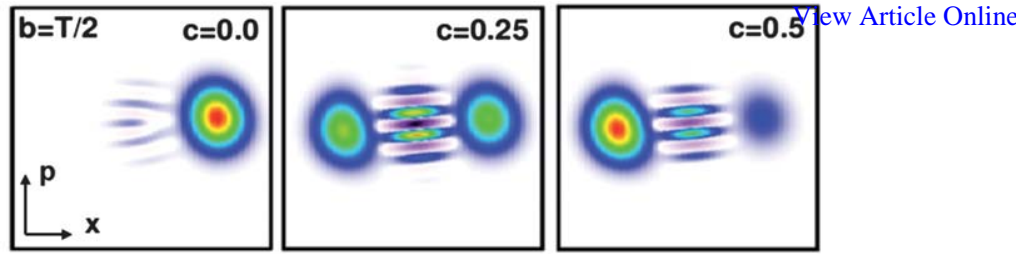

(b)
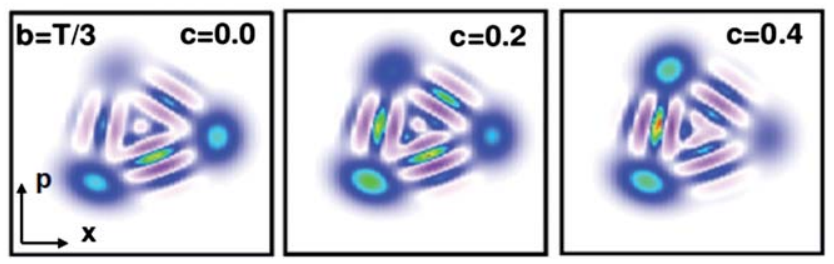

Fig. 5 Simulation of wavepackets in Wigner representation and its dependence on the subpulse separation (parameter $b$ ) and their relative phase (parameter $c$ ). (a) Wavepacket for a sub-pulse separation equal to half of the period of low-frequency molecular mode. (b) Wavepacket for a sub-pulse separation equals to one third of the period of low-frequency molecular mode.

wavepacket, the choice of $b=(\beta / \alpha) T_{\text {vib }}(\alpha, \beta \in \mathbb{N})$ leads to the formation of $\alpha$ subwavepackets, phase shifted by $2 \pi \times \beta / \alpha .{ }^{24}$ The maximum value of $\alpha$ is limited by the number of vibrational levels of the coupling mode contributing to the excited wavepacket. Fig. 5 shows two examples of these wavepackets in the Wigner representation for $b=T_{\mathrm{vib}} / 2$ and $T_{\mathrm{vib}} / 3$.

The generated phase space structures in the dynamically active mode imprint themselves in the population transfer between $S_{2}$ and $S_{1}$. Analogously to the experimental observations, we observe tailored population decay depending on the parameter $c$. This is shown in Fig. 4 (e) and (f). By choosing the same experimental values, i.e. $b=56$ fs and $c=0 \mathrm{rad}$ and $c=2.3 \mathrm{rad}$, the same oscillatory component and its dependence on the relative phase between the sub-pulses can be perfectly simulated. In perfect agreement with the experimental results (Fig. 4), the electronic coupling manifests itself in a blue shift from $178 \mathrm{~cm}^{-1}$ for the uncoupled mode, to $200 \mathrm{~cm}^{-1}$. The Fourier transformation of this oscillatory component has also a maximum for $c=0 \mathrm{rad}$, while for $c=2.3 \mathrm{rad}$ it can be almost suppressed. The wavepacket generated in the case of $b=56$ fs corresponds approximately to $T_{\mathrm{vib}} / 3$ of the electronically coupled mode and leads to the generation of three sub-wavepackets in the $\mathrm{S}_{2}$ potential surface, i.e. $\alpha=3$.

Contrasting to the experiment and its inherent constraints, the simulation is also able to investigate directly the transient vibrational population during the multipulse excitation. This description is very insightful since it explains the relative time dependent weight of the $\mathrm{S}_{2}$ vibrational states constituting the wavepacket during the pulse train excitation. A generalization of the described observations to any choice of $\alpha$ in the valid range is possible. For simplicity, we discuss the simplest case of $b=T_{\mathrm{vib}} / 2$, hence $\alpha=2$. For $b=T_{\text {vib }} / 2$, we observe that vibrational states with odd quantum numbers are much less sensitive to the phase parameter $c$. The population of the vibrational levels $v=0$ up to $v=4$ is compared in Fig. 6 (a) and (b) for two values of $c$ (1.4 rad and $1.75 \mathrm{rad}$, respectively). Their population follows a Gaussian distribution, initially induced by the first sub-pulse. While scanning $c$, we observe an oscillatory behaviour of the vibrational populations with a periodicity of nearly $2 \pi /(2 \alpha)$, i.e. twice the number of sub-wavepackets. The maximum asymmetry in distribution is obtained intermediately in the second half of the pulse train, as indicated by the dotted lines in Fig. 6 (c) and (d). In this case, depending on $c$, the even vibrational states are either enhanced or depopulated by the sub-pulse following the central 

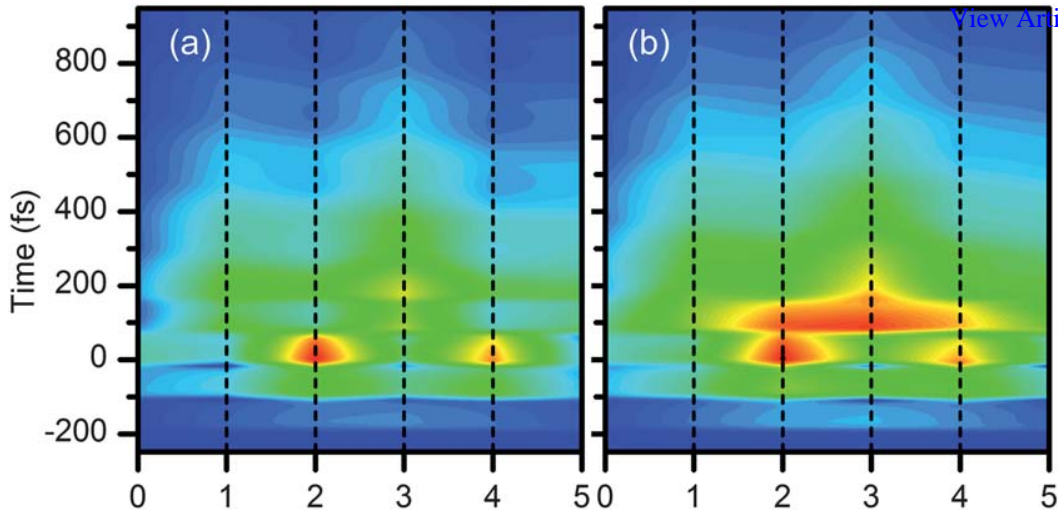

Vibrational Level

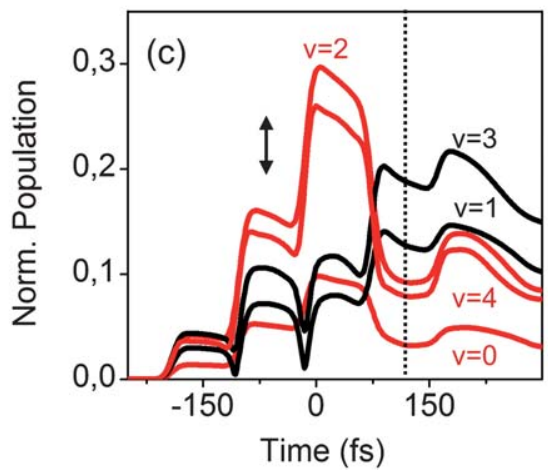

Vibrational Level

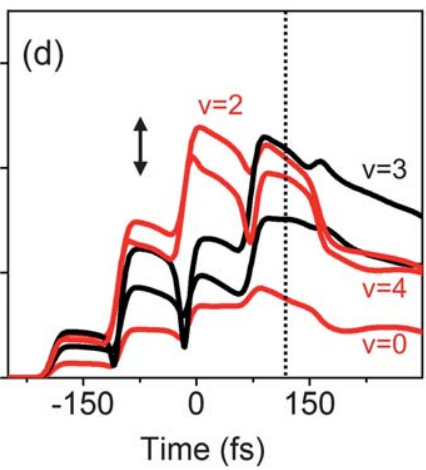

Fig. 6 Simulation of the transient evolution of the vibrational population and its dependence on parameter $c$ for $b=T_{\mathrm{vib}} / 2$. (a) and (b) show the vibrational population for $c=1.4 \mathrm{rad}$ and $1.75 \mathrm{rad}$, respectively. (c) and (d) show the same data but with an individual curve for each vibrational level. Time zero defines the centre of multipulse excitation. Red curves are associated with even vibrational levels $(v=0,2$ and 4$)$, while black dashed curves are related to odd vibrational levels ( $v=1$ and 3 ). The difference in the evolution of even vibrational levels in dependence of $c$ can be clearly observed, e.g., at about $100 \mathrm{fs}$ (dotted lines).

one, imprinting holes in the overall Gaussian distribution. We observe maxima of FFT amplitudes for $c=2 n \pi /(2 \alpha), n \in \mathbb{N}$ and minima at $c=(2 n+1) \pi /(2 \alpha)$.

\section{Discussion}

\section{Understanding the control mechanism}

In order to rationalize the control mechanism, two points must be discussed. The first is related to the effect of multipulse excitation on wavepackets. Our simulations show that the interference between wavepackets generated by different sub-pulses depends on their relative phase. Of course, this interference, which can be constructive or destructive, is not equal for all vibrational levels on the excitation bandwidth of the laser since each level has different time evolution factors. This can be clearly observed by comparing Fig. 6 (c) and (d): By changing the relative phase between the sub-pulses, a different transient evolution for each vibrational level can be tailored.

The second point is related to the interaction of this transient vibrational population with a non-adiabatic coupling. In general, the degree of coupling between two electronic surfaces is not necessarily homogeneous but depends on the vibrational 

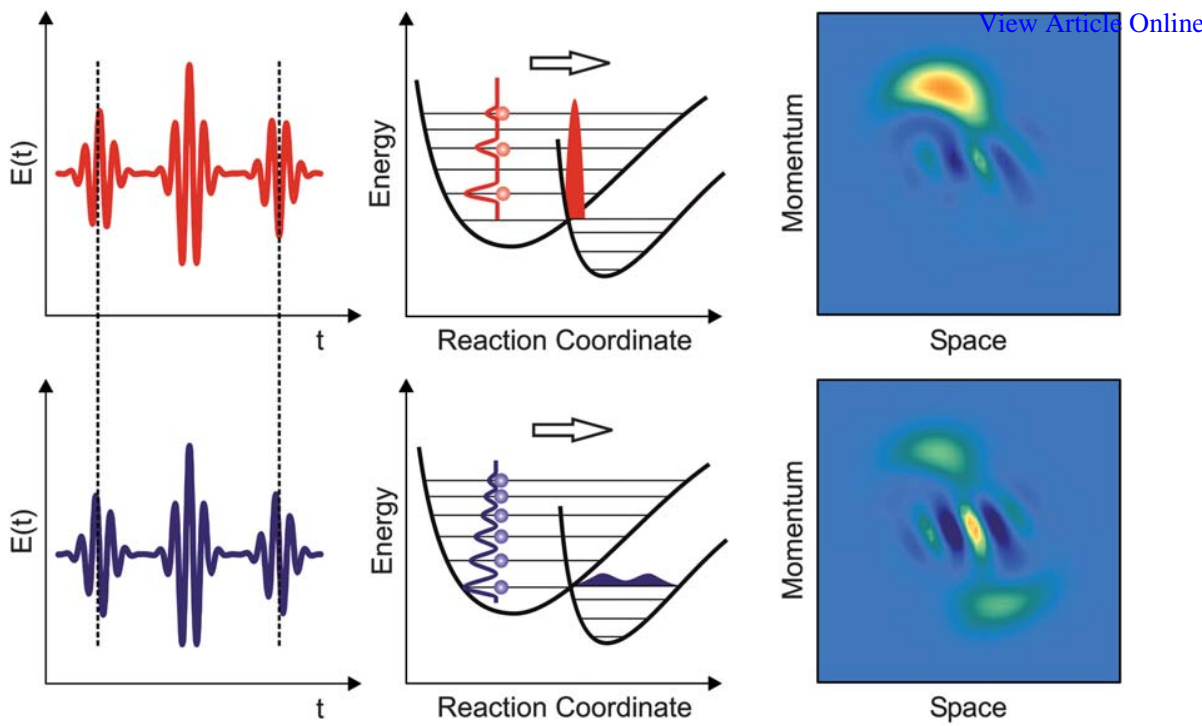

Fig. 7 Effect of the relative sub-pulse phase for the case of $b=T_{\mathrm{vib}} / 2(\alpha=2)$ on the composition of the resulting wavepacket in the vicinity of the CoIn. The left column shows the excitation pulse trains with differing parameter $c$, the middle column shows a scheme of the vibrational populations of the excited wavepacket after the sub-pulse following the central one and the resulting wavepacket, the right column shows the wavepacket in the phase space representation of the coupling mode. In the upper row a properly spaced pulse train creates a wavepacket, composed mainly of odd wavefunctions. The pulses in lower and upper rows differ by a relative phase shift of $\pi / 4$. The blue pulse train yields a delocalized wavepacket with evenly distributed eigenstate-coefficients. In the phase space representation we observe the phase shift of $\pi$ between the two components consisting of odd and even eigenstates and an interference pattern in between.

level. In other words, the magnitude of the vibronic coupling matrix elements depends on the vibrational quantum number in the respective coupling mode. If in such a scenario a wavepacket is prepared which lacks contributions with strong coupling elements, ${ }^{29,30}$ the overall non-adiabatic transition will be less effective.

These two points are summed up in Fig. 7. We depict the excitation pulse trains and their relative phase differences (left column; see the shape of the electric fields), the distribution of the $S_{2}$ vibrational states after the sub-pulse following the central one (middle column) and the Wigner representation of the corresponding wavepackets after the pulse train (right column). The top row represents the scenario for subpulse spacing $b=T_{\mathrm{vib}} / 2$ and relative phase $c=2 n \pi / 4$. In the vibrational distribution (middle column) we observe holes at the even levels as discussed above. The annihilation of the corresponding sub-wavepackets, visible in the Wigner representation (right column), leads to the formation of a sharp FFT peak. Therefore, the experimental data in Fig. 4 (c), obtained for $c=0$, can be assigned to the related scenario with $\alpha=3$. The lower row in Fig. 7 shows the reversed case for $c=(2 n+1) \pi / 4$, where the vibrational population follows again a Gaussian distribution. The appearance of two phase shifted sub-wavepackets leads to the depletion of the FFT-signal. This case again is in good agreement to the experimental data shown in Fig. 4 (f), where for the case of $\alpha=3$ and $n=2$ the formation of three wavepackets leads to the observed depletion.

\section{Extending the control mechanism to competing channels}

The transient tailoring of vibrational population should ultimately affect the electronic lifetime of the $S_{2}$ state, i.e., the internal conversion between $S_{2}-S_{1}$ states. In 
this situation, the population will remain longer in the higher lying electroaic state Online compared to Fourier-limited excitation. Not surprisingly, the tailoring of wavepackets does not affect the energy flow in $\beta$-carotene, since the excited state population experiences just one effective deactivation channel i.e. via $\mathrm{S}_{1}$ state. In this regard it can be considered as a closed system, where control via one-photon excitation is not possible. ${ }^{31,32}$ Phase control with one-photon transition is, however, possible if strong dissipative coupling (with e.g. the environment) takes place and assists the control mechanism, ${ }^{33}$ as has been demonstrated to constrain the control of population transfer from the ground- to the excited-state in closed systems. ${ }^{17,18,34}$

In the case of open systems with competing channels, like those found in photosynthetic systems, the branching ratio may be directly affected via one-photon phase control. ${ }^{33}$ In that sense, our findings are able to rationalize pulse shapes found in the coherent control of energy flow in polyenic biochromophores, where the excited state lifetime is at the order of the total duration of the excitation pulse train. A prerequisite for the mechanism proposed here is a low frequency mode coupling the initially excited state and its successor. This criterion is met by many large and medium sized organic molecules and is already confirmed by theory ${ }^{35}$ for polyenes. Besides our explanation does not only describe the findings in bacteriorhodopsin, ${ }^{15,29}$ but also provides a commonly applicable mechanism for the control of ultrafast photoisomerizations, where the decisive conical intersection lies in the vicinity of an excited state minimum. ${ }^{36}$

Moreover, the good agreement of our experimental data with the simulations unravels a new feature of carotenoid's ultrafast photochemistry. The early photochemistry of carotenoids is determined by strong vibronic coupling like that given by a conical intersection (CoIn). By the aid of coherent control and quantum dynamical modelling, through the observed phase dependencies, we reveal the matter wave character of the process, even preserved through the CoIn, and identify an effective low-frequency coupling mode of $200 \mathrm{~cm}^{-1}$ driving this ultrafast internal conversion process. Such out-of-plane backbone motion in the carotenoid as part of a Light harvesting complex (LHC) has been discussed as the source of conformational change affecting the biological function. ${ }^{9,35}$

\section{Conclusion}

In this paper we addressed for the first time a general mechanism to explain the control of energy flow reported for a whole class of biochromophores. In particular, we show how a specifically designed light pulse tailors the induced wavepacket dynamics. By a detailed and accurate simulation of our results, we identify the nuclear degree of freedom which couples the two relevant excited electronic states in the investigated chromophore. Our control mechanism is based on the interference between wavepackets in the excited state, which leads to a transient evolution of the vibrational population dependent on the relative phase between excitation sub-pulses. Such a transient evolution can affect the branching ratio between competing channels in the excited state. Thus, we are able to rationalize pulse shapes found in a whole class of coherent control experiments involving polyenic biochromophores, like in light harvesting complexes and bacteriorhodopsin. Furthermore, we show that the coherence imprinted by the pulse train is preserved through the conical intersection pointing out how coherence could be exploited to manipulate the energy transfer process in these systems.

We note that in the present study, the use of coherent control goes beyond the scope of product optimization demonstrated in numerous studies. ${ }^{37,38}$ In several works, light was used as a photonic reagent, ${ }^{39,40}$ catalys $^{41,42}$ or as tomographic tool. ${ }^{43}$ The present investigation provides a case where tailored light pulses are employed as a highly specialized diagnostic tool for photophysical processes impossible to describe by the use of unmodulated light pulses. In contrast to conventional 
coherent control, we would like to call this approach Quantum Controlvspectroste Online copy (QCS).

\section{References}

1 R. Blankenship, Molecular Mechanisms of Photosynthesis, Blackwell Publishers, Malden, MA, 2002.

2 H. A. Frank, A. J. Young, G. Britton and R. J. Cogdell, ed., The Photochemistry of Carotenoids, Kluwer Academic Publishers, Govindjee, 1999.

3 T. Polivka and V. Sundstrom, Chem. Rev., 2004, 104, 2021-2071.

4 T. Buckup, J. Savolainen, W. Wohlleben, J. L. Herek, H. Hashimoto, R. R. B. Correia and M. Motzkus, J. Chem. Phys., 2006, 125, 194505.

5 A. Ishizaki, T. R. Calhoun, G. S. Schlau-Cohen and G. R. Fleming, Phys. Chem. Chem. Phys., 2010, 12, 7319-7337.

6 C. J. Bardeen, V. V. Yakovlev, J. A. Squier and K. R. Wilson, J. Am. Chem. Soc., 1998, 120, 13023-13027.

7 P. F. Tian, D. Keusters, Y. Suzaki and W. S. Warren, Science, 2003, 300, 1553-1555.

8 D. Abramavicius and S. Mukamel, J. Chem. Phys., 2004, 120, 8373-8378.

9 W. Wohlleben, T. Buckup, J. L. Herek and M. Motzkus, ChemPhysChem, 2005, 6, 850-857.

10 T. Buckup, T. Lebold, A. Weigel, W. Wohlleben and M. Motzkus, J. Photochem. Photobiol., A, 2006, 180, 314-321.

11 G. Vogt, P. Nuernberger, T. Brixner and G. Gerber, Chem. Phys. Lett., 2006, 433, $211-$ 215.

12 D. G. Kuroda, C. P. Singh, Z. H. Peng and V. D. Kleiman, Science, 2009, 326, 263-267.

13 J. L. Herek, W. Wohlleben, R. J. Cogdell, D. Zeidler and M. Motzkus, Nature, 2002, 417, 533-535.

14 J. Savolainen, R. Fanciulli, N. Dijkhuizen, A. L. Moore, J. Hauer, T. Buckup, M. Motzkus and J. L. Herek, Proc. Natl. Acad. Sci. U. S. A., 2008, 105, 7641-7646.

15 V. I. Prokhorenko, A. M. Nagy, S. A. Waschuk, L. S. Brown, R. R. Birge and R. J. D. Miller, Science, 2006, 313, 1257-1261.

16 A. M. Weiner, D. E. Leaird, G. P. Wiederrecht and K. A. Nelson, Science, 1990, 247, $1317-$ 1319.

17 J. Hauer, T. Buckup and M. Motzkus, J. Chem. Phys., 2006, 125, 061101.

18 T. Buckup, J. Hauer, C. Serrat and M. Motzkus, J. Phys. B: At., Mol. Opt. Phys., 2008, 41, 074024.

19 J. Oberle, G. Jonusauskas, E. Abraham and C. Rulliere, Chem. Phys. Lett., 1995, 241, $281-$ 289.

20 M. Motzkus, S. Pedersen and A. H. Zewail, J. Phys. Chem., 1996, 100, 5620-5633.

21 T. Hornung, H. Skenderovic and M. Motzkus, Chem. Phys. Lett., 2005, 402, $283-288$.

22 J. Hauer, T. Buckup and M. Motzkus, J. Phys. Chem. A, 2007, 111, 10517-10529.

23 T. Buckup, J. Hauer, J. Mohring and M. Motzkus, Arch. Biochem. Biophys., 2009, 483, 219 223.

24 J. Voll and R. de Vivie-Riedle, New J. Phys., 2009, 11, 105036.

25 T. Polivka and V. Sundstrom, Chem. Phys. Lett., 2009, 477, 1-11.

26 D. Zeidler, S. Frey, K.-L. Kompa and M. Motzkus, Phys. Rev. A: At., Mol., Opt. Phys., 2001, 64, 3421-3433.

27 J. Hauer, H. Skenderovic, K. L. Kompa and M. Motzkus, Chem. Phys. Lett., 2006, 421, 523-528.

28 T. Buckup, J. Hauer and M. Motzkus, New J. Phys., 2009, 11, 105049.

29 V. I. Prokhorenko, A. M. Nagy, L. S. Brown and R. J. D. Miller, Chem. Phys., 2007, 341, 296-309.

30 P. S. Christopher, M. Shapiro and P. Brumer, J. Chem. Phys., 2006, 125, 124310.

31 P. Brumer and M. Shapiro, Chem. Phys., 1989, 139, 221-228.

32 M. Shapiro and P. Brumer, Principles of the Quantum Control of Molecular Processes, John Wiley \& Sons, Hoboken, 2003.

33 M. Spanner, C. A. Arango and P. Brumer, J. Chem. Phys., 2010, 133.

34 V. I. Prokhorenko, A. M. Nagy and R. J. D. Miller, Journal of Chemical Physics, 2005, 122.

35 W. Fuss, Y. Haas and S. Zilberg, Chem. Phys., 2000, 259, 273-295.

36 B. Dietzek, B. Bruggemann, T. Pascher and A. Yartsev, Phys. Rev. Lett., 2006, 97, 258301.

37 C. J. Bardeen, V. V. Yakovlev, K. R. Wilson, S. D. Carpenter, P. M. Weber and W. S. Warren, Chem. Phys. Lett., 1997, 280, 151-158.

38 A. Assion, T. Baumert, M. Bergt, T. Brixner, B. Kiefer, V. Seyfried, M. Strehle and G. Gerber, Science, 1998, 282, 919-922.

39 H. Rabitz, Science, 2003, 299, 525-527. 
40 C. Daniel, J. Full, L. Gonzalez, C. Lupulescu, J. Manz, A. Merli, S. Vajda andilewWXøstede Online Science, 2003, 299, 536-539.

41 B. J. Sussman, D. Townsend, M. Y. Ivanov and A. Stolow, Science, 2006, 314, 278-281.

42 R. J. Levis, G. M. Menkir and H. Rabitz, Science, 2001, 292, 709-713.

43 M. Wollenhaupt, M. Krug, J. Kohler, T. Bayer, C. Sarpe-Tudoran and T. Baumert, Appl. Phys. B: Lasers Opt., 2009, 95, 647-651. 